Original Article

\title{
The influence of unstable modified wall squat exercises on the posture of female university students
}

\author{
Yoonmi Lee, $\mathrm{PhD}, \mathrm{PT}^{1)}$ \\ 1) Department of Occupational Therapy, Gumi University: 407 Bugok-dong, Gumi-si, \\ Gyeongsangbukdo 730-711, Republic of Korea
}

\begin{abstract}
Purpose] The purpose of this study was to examine the effect of unstable modified wall squat exercises on the posture of female university students. [Subjects] The subjects of this study were 30 female university students who were equally and randomly allocated to an unstable modified wall squat exercises group the experimental group and a stable modified wall squat exercises group the control group. [Methods] Both groups performed their respective exercises for 30 minutes three times per week over a six-week period. Using BackMapper, trunk inclination, trunk imbalance, pelvic position, pelvic torsion, pelvic rotation, and position of the scapulae were evaluated. [Results] The unstable modified wall squat exercises group obtained significant results for trunk inclination, trunk imbalance, pelvic position, pelvic torsion, position of the scapulae, while the stable modified wall squat exercises group obtained significant results for trunk imbalance and pelvic position. [Conclusion] Unstable modified wall squat exercises may be applied as a method to correct the posture of average adults.

Key words: Wall squat exercises, BackMapper, Posture
\end{abstract}

(This article was submitted Apr. 2, 2015, and was accepted Apr. 24, 2015)

\section{INTRODUCTION}

Trunk muscles, which are important in maintaining posture, can be largely divided into two groups: motor muscles and local stabilizers. Motor muscles, such as the erector spinae and rectus abdominis, are involved in movement and load carrying between the spine and the pelvis. Local stabilizers, such as the transversus abdominis and multifidus, play a role in stabilizing the spine during postural movements when a weight or load is placed on the spinal structure ${ }^{1)}$. Body trunk stabilization exercise is generally used for stabilization of the spine and pelvis, are important for maintaining posture. The purpose of body trunk stabilization is to increase the stability of the spine and pelvis, reinforce muscle strength, and restore muscle and balance control ${ }^{2}$. Muscles that are activated in body trunk stabilization exercises include the transversus abdominis, internal obliques, external obliques, quadratus lumborum, multifidus, and pelvic floor muscles. Multifidus muscles and the transversus abdominis, in particular, control body trunk balance, as they are stimulated before other muscles when the body moves. The bilateral internal abdominal obliques are also important in maintaining the spine's lateral stability and flexion ability ${ }^{3)}$. Exces-

Corresponding author. Yoonmi Lee (E-mail: yoonmipt@ hanmail.net)

(C2015 The Society of Physical Therapy Science. Published by IPEC Inc. This is an open-access article distributed under the terms of the Creative Commons Attribution Non-Commercial No Derivatives (by-ncnd) License $<$ http://creativecommons.org/licenses/by-nc-nd/3.0/>. sive lumbar lordosis or pelvic anterior tilt can occur during the modified wall squat exercise. The abdominal drawing-in technique can reduce the occurrence of this by inducing simultaneous subtraction of the transversus abdominis and internal abdominal oblique muscles during the trunk stabilization part of the exercise ${ }^{4)}$.

Squat exercises focus on developing both lower and upper body muscles ${ }^{5}$, and they can be performed relatively easily in any setting. Hence, they are commonly used in many sports to enhance athletic performance, as well as in postsurgery rehabilitation programs ${ }^{6}$. Despite the many advantages of squat exercises, there is a potential risk than an unstable posture during the exercise could cause damage to the lower back or place undue pressure on the knees ${ }^{7}$. Wall squat exercises are a modified version of squat exercises aimed at overcoming these potential risks. To prevent possible lower back or knee damage, the subject supports his/her body weight against the wall while squatting. This wall squat exercise is easy for beginners to perform. The modified wall squat exercise in the present study puts more emphasis on lumbar stability than on reinforcement of lower body muscle strength by including motions aimed at reinforcing neck and shoulder stability.

Recent studies of body trunk stabilization exercise have utilized a variety of tools, such as unstable ground, balancing boards, therapy balls, and sponge pads ${ }^{8}$. O'Sullivan et al. argued that stabilization exercise can maximize the improvement in balancing ability when it is performed in a dynamic environment, such as on a therapy ball, rather than in a static environment ${ }^{9}$ ). Page also suggested that exercises using unstable tools are effective for postural maintenance or 
somesthesia recovery ${ }^{10}$.

Although the effects of the modified wall squat exercise on deep abdominal muscle thickness and lumbar stability have been studied ${ }^{11)}$, no study has specifically examined the impact of a modified wall squat exercise executed on an unstable support surface. Therefore, the present study investigated the effect of a modified wall squat exercise performed on an unstable support surface on posture. The aim of the exercise was stimulation of somatosensory receptors.

\section{SUBJECTS AND METHODS}

Thirty female students enrolled in college at the time of the study were selected as the sample. They were randomly and evenly divided into an experimental group that performed unstable modified wall squat exercises and a control group that performed stable modified wall squat exercises.

The selection criteria were as follows: no structural abnormality of the spine before participation in the experiment, no pain in the spine (such as low back pain), no medication, no neurological diseases, no drinking during the experiment, and not overweight. Those who performed weight training, which can affect posture, and those who performed regular exercise were excluded from the experiment. This study was approved by university's institutional review board, and the safety of the subjects was protected during all of the processes of the experiment. All of the subjects understood the purpose of this study and provided written informed consent prior to participation in the study in accordance with the ethical standards of the Declaration of Helsinki.

The age, height, and weight of the participants in the experimental group were $21.6 \pm 3.6$ years, $158.0 \pm 6.3 \mathrm{~cm}$, and $52.7 \pm 3.1 \mathrm{~kg}$, respectively. Those in the control group were $22.2 \pm 4.3$ years, $157.4 \pm 5.6 \mathrm{~cm}$, and $51.3 \pm 5.5 \mathrm{~kg}$, respectively. A $\chi^{2}$ test was used for analysis of gender, and an independent $t$-test was used for the analysis of age, height, and body weight. According to the results of the analysis, which showed no statistically significant differences $(p>0.05)$, homogeneity was not a problem between the two groups.

In this study, the subjects in the experimental group performed the modified wall squat exercise on an unstable support surface using the abdominal drawing-in technique. The control group performed the exercise on stable ground. An Airex Balance Pad (Alcan-Airex AG, Sins, Switzerland) with a width of $50 \mathrm{~cm}$, length of $41 \mathrm{~cm}$, and height of $6 \mathrm{~cm}$ was used as an unstable support surface while performing the modified wall squat exercise with the abdominal drawing-in technique.

Abdominal drawing-in can decrease excessive lumbar lordosis or pelvic anterior tilt by inducing simultaneous subtraction of the transversus abdominis and internal obliques during lumbar stability exercise ${ }^{4}$.

For the modified wall squat exercises, the subjects stood about a foot from a wall with their legs spread shoulder-width apart. With their fingers spread out, one hand was held with the palm end upward from the chest, while the finger ends of the other hand around the belly were held downward, and pulled the chin was pulled in pushing the head toward the wall. The pelvis and the lumbar spine were in the neutral position. During the squat motion, the soles of the feet did not leave the ground, the knees were flexed up to 90 degrees, where they remained for 5 seconds, and then the knees were extended back to 10 degrees which was maintained for 3 seconds ${ }^{11)}$. One set consisted of 10 repetitions of the movements described above, and the subjects had a 15 -sec of rest between each set. Ten sets were performed $15 \mathrm{sec}$ of $30 \mathrm{~min}$, 3 days a week, for 6 weeks.

A three-dimensional spine diagnosis system (BackMapper, ABW, Frickenhausen, Germany) was used to measure postural change. The BackMapper system relatively accurately measures and analyzes the shape and location of the spine and the severity of pelvic distortion. It provides front and back images of the spine, as well as images from above, beneath, and the left and right. The device provides data on trunk inclination (TIN), which indicates the spine's inclination in a sagittal plane; trunk imbalance (TIM), which indicates the spine's inclination in a coronal plane; pelvic position (PPO), which indicates horizontal tilt of the pelvis; pelvic torsion (PTO), which indicates the extent of hip bone rotation; pelvic rotation (PRO), which indicates the rotation of the pelvis in a horizontal plane; the position of the scapulae (PSA), which indicates the height of the left and right shoulder blades. The system also analyses the distribution of muscle and fat across the body, as well as the location of the body frame, using diverse variables.

The measurement data were analyzed using the statistical program SPSS 12.0 KO (SPSS, Chicago, IL, USA). The mean and standard deviation of the data are presented. A paired t-test was used for verifying the significance of individual group differences before and after the experiment, and an independent $t$-test was used for verifying the significance of between-group differences. The significance level $\alpha$ was set at 0.05 .

\section{RESULTS}

The results revealed a statistically significant difference in TIN, TIM, PPO, PTO, and PSA in the experimental group after the intervention. In contrast, only the TIM and PPO showed a statistically difference in the control group after the intervention $(\mathrm{p}<0.05)$ (Table 1). We compared the values of the two groups before and after the intervention and the changes in their values between before and after the intervention. We found no statistically significant difference between the groups before the intervention and no statistically significant change in values between the two groups after the intervention. Statistical significance was observed after the intervention for TIN, PTO, and PSA $(\mathrm{p}<0.05)$ (Table 2).

\section{DISCUSSION}

Correct posture, which refers to the ideal musculoskeletal alignment, affects not only the functional efficiency of the body but also a person's appearance. The pelvis is of central importance for correct posture. The pelvis supports the abdomen and connects the spine to the lower extremities. In a standing position, it delivers body weight from the spine to the lower extremities and maintains a correct posture, enabling liberal movement of the upper extremities ${ }^{12}$. Trunk stability exercise is receiving increasing attention today 
Table 1. Comparison of TIN, TIM, PPO, PTO, PRO, and PSA between before and after the intervention in each group (mean \pm SD) (units: TIN, TIM, PPO, PTO, and PRO degree; PSA, mm)

\begin{tabular}{llcc}
\hline Category & Group & $\begin{array}{c}\text { Before } \\
\text { intervention }\end{array}$ & $\begin{array}{c}\text { After } \\
\text { intervention }\end{array}$ \\
\hline \multirow{2}{*}{ TIN } & Experimental group* & $2.6 \pm 1.5$ & $1.2 \pm 0.8$ \\
& Control group & $2.7 \pm 1.2$ & $2.0 \pm 1.0$ \\
\hline \multirow{2}{*}{ TIM } & Experimental group* & $2.9 \pm 1.2$ & $1.3 \pm 0.7$ \\
& Control group* & $2.8 \pm 1.5$ & $1.5 \pm 1.0$ \\
\hline \multirow{2}{*}{ PPO } & Experimental group* & $2.7 \pm 1.4$ & $1.2 \pm 0.9$ \\
& Control group* & $2.5 \pm 1.4$ & $1.5 \pm 0.8$ \\
\hline \multirow{2}{*}{ PTO } & Experimental group* & $3.5 \pm 1.9$ & $1.4 \pm 0.6$ \\
& Control group & $3.4 \pm 2.9$ & $2.4 \pm 1.2$ \\
\hline \multirow{2}{*}{ PRO } & Experimental group & $2.7 \pm 2.2$ & $1.5 \pm 0.7$ \\
& Control group & $2.7 \pm 1.8$ & $2.2 \pm 1.4$ \\
\hline \multirow{2}{*}{ PSA } & Experimental group* & $5.5 \pm 2.9$ & $2.8 \pm 1.2$ \\
& Control group & $5.3 \pm 2.5$ & $3.6 \pm 2.2$ \\
\hline
\end{tabular}

$* \mathrm{p}<0.05$. TIN: trunk inclination; TIM: trunk imbalance; PPO pelvic position; PTO: pelvic torsion; PRO: pelvic rotation; PSA: position of scapulae

due to its effectiveness in postural control. Trunk stability exercise training focuses on the pelvis, which contributes to the balanced activation of the abdominal muscles and back muscles, which play an important role in maintaining stability and controlling body trunk posture ${ }^{13)}$. Kinesthetic senses are essential in recognizing correct alignment of the trunk of the body ${ }^{14}$. These senses are largely influenced by sensory inputs from sensory receptors distributed throughout the skin, muscle, and joints ${ }^{14)}$. Correct posture is very important in activities of daily living. The literature includes a number of studies reporting changes in deep abdominal muscle thickness and lumbar stability after trunk stability exercise or instant posture change after pelvic correction. However, there have been no previous studies of the impact on posture of modified wall squat exercises on an unstable support surface aimed at stimulating the sensory receptors. Hence, the present study investigated the effect of unstable modified wall squat exercises on the posture of female college students.

Lee et al. performed selective trunk stability and lumbar exercises targeting two groups of deep abdominal muscles for 5 weeks ${ }^{15}$. They reported that both exercises were effective in increasing deep abdominal muscle thickness and argued that such exercises are essential for patients who require posture control for lumbar stability improvement. Endleman measured the muscle thickness of the transversus abdominis and internal oblique abdominis following Pilates core training in 18 female adults and 8 male adults for 6 months ${ }^{16)}$. They found a significant difference in the muscle thickness of the transversus abdominis, indicating a positive impact of core training on reinforcement of abdominal muscles and body trunk stabilization. Brill reported that core stabilization exercise was effective in enhancing muscle strength and lumbar stability because it maintained spinal balance due to the contraction of the transverse muscle of
Table 2. Comparison of TIN, TIM, PPO, PTO, PRO, and PSA between the experimental group and control group (mean $\pm \mathrm{SD}$ ) (units: TIN, TIM, PPO, PTO, and PRO degree; PSA, mm)

\begin{tabular}{lccc}
\hline & Category & $\begin{array}{c}\text { Experimental } \\
\text { group }\end{array}$ & $\begin{array}{c}\text { Control } \\
\text { group }\end{array}$ \\
\hline & TIN & $2.6 \pm 1.5$ & $2.7 \pm 1.2$ \\
Before & TIM & $2.9 \pm 1.2$ & $2.8 \pm 1.5$ \\
intervention & PPO & $2.7 \pm 1.4$ & $2.5 \pm 1.4$ \\
& PTO & $3.5 \pm 1.9$ & $3.4 \pm 2.9$ \\
& PRO & $2.7 \pm 2.2$ & $2.7 \pm 1.8$ \\
& PSA & $5.5 \pm 2.9$ & $5.3 \pm 2.5$ \\
\hline \multirow{3}{*}{ After } & TIN* & $1.2 \pm 0.8$ & $2.0 \pm 1.0$ \\
& TIM & $1.3 \pm 0.7$ & $1.5 \pm 1.0$ \\
& PPO & $1.2 \pm 0.9$ & $1.5 \pm 0.8$ \\
& PTO* & $1.4 \pm 0.6$ & $2.4 \pm 1.2$ \\
& PRO & $1.5 \pm 0.7$ & $2.2 \pm 1.4$ \\
& PSA* & $2.8 \pm 1.2$ & $3.6 \pm 2.2$ \\
\hline \multirow{2}{*}{ bhange between } & TIN & $1.4 \pm 1.9$ & $0.7 \pm 1.4$ \\
intervention & PPO & $1.5 \pm 1.5$ & $1.2 \pm 2.1$ \\
& PTO & $2.0 \pm 2.1$ & $1.0 \pm 3.6$ \\
& PRO & $1.1 \pm 2.4$ & $0.5 \pm 1.4$ \\
& PSA & $2.7 \pm 3.0$ & $1.7 \pm 3.6$ \\
\hline \multirow{2}{*}{ and after } & & &
\end{tabular}

$* \mathrm{p}<0.05$

the abdomen ${ }^{17)}$.

Cho evenly divided 30 female college students into a pelvic adjustment group and a stretching group that performed pelvic adjustments using the Gonstead technique and examined the effect on postural change ${ }^{18)}$. The results showed that the exercise had a positive impact on postural change. In a study involving modified wall squat exercises, 30 female college students were evenly divided into a modified wall squat exercise group and a bridge exercise on a stable support surface group ${ }^{11)}$. The study reported that the modified wall squat exercise had positive effects on deep abdominal muscle thickness and lumbar stability.

Comparing the results before the intervention and after the intervention in the present study, TIN, TIM, PPO, PTO, and PSA showed a statistically significant difference in the experimental group. In contrast, the control group showed a statistically significant difference only in TIM and PPO. This finding can be attributed to the unstable modified wall squat exercise, together with abdominal drawing-in, activating the deep muscles related to maintaining posture. Performing the wall squat exercise while trying to maintain the stability of the body trunk on an unstable support surface enhanced postural changes compared with performing the modified wall squat exercise on a stable support surface.

In summary, the modified wall squat stabilization exercise, in particular, unstable modified wall squat exercises with abdominal drawing-in, can be recommended for correction of posture. 


\section{REFERENCES}

1) Standaert CJ, Weinstein SM, Rumpeltes J: Evidence-informed management of chronic low back pain with lumbar stabilization exercises. Spine J, 2008, 8: 114-120. [Medline] [CrossRef]

2) Richardson CA, Snijders CJ, Hides JA, et al.: The relation between the transversus abdominis muscles, sacroiliac joint mechanics, and low back pain. Spine, 2002, 27: 399-405. [Medline] [CrossRef]

3) O'Sullivan PB, Grahamslaw KM, Kendell M, et al.: The effect of differen standing and sitting postures on trunk muscle activity in a pain-free population. Spine, 2002, 27: 1238-1244. [Medline] [CrossRef]

4) Willson JD, Dougherty CP, Ireland ML, et al.: Core stability and its relationship to lower extremity function and injury. J Am Acad Orthop Surg, 2005, 13: 316-325. [Medline]

5) Goldberg L, Elliot DL, Kuehl KS: A comparison of the cardiovascular effects of running and weight training. J Strength Cond Res, 1996, 20: 219-224.

6) Stuart MJ, Meglan DA, Lutz GE, et al.: Comparison of intersegmental tibiofemoral joint forces and muscle activity during various closed kinetic chain exercises. Am J Sports Med, 1996, 24: 792-799. [Medline] [CrossRef]

7) Fry AC: Coaching considerations for the barbell squat-Part 1. J Strength Cond Res, 1993, 15: 556-569.

8) Arokoski JP, Valta T, Airaksinen O, et al.: Back and abdominal muscle function during stabilization exercises. Arch Phys Med Rehabil, 2001, 82: 1089-1098. [Medline] [CrossRef]

9) O'Sullivan PB, Phyty GD, Twomey LT, et al.: Evaluation of specific stabilizing exercise in the treatment of chronic low back pain with radiologic di- agnosis of spondylolysis or spondylolisthesis. Spine, 1997, 22: 2959-2967. [Medline] [CrossRef]

10) Page P: Sensorimotor training: A "global" approach for balance training. J Bodyw Mov Ther, 2006, 10: 77-84. [CrossRef]

11) Cho M: The effects of modified wall squat exercises on average adults' deep abdominal muscle thickness and lumbar stability. J Phys Ther Sci, 2013, 25: 689-692. [Medline] [CrossRef]

12) Kapandji IA: Physiology of the Joints, 6th ed. Philadelphia: Churchill Livingstone. 2007

13) Marshall PW, Murphy BA: Evaluation of functional and neuromuscular changes after exercise rehabilitation for low back pain using a Swiss ball: a pilot study. J Manipulative Physiol Ther, 2006, 29: 550-560. [Medline] [CrossRef]

14) Maffey-Ward L, Jull G, Wellington L: Toward a clinical test of lumbar spine kinesthesia. J Orthop Sports Phys Ther, 1996, 24: 354-358. [Medline] [CrossRef]

15) Lee JS, Kim TH, Kim DY, et al.: Effects of selective exercise for the deep abdominal muscles and lumbar stabilization exercise on the thickness of the transversus abdominis and postural maintenance. J Phys Ther Sci, 2015, 27: 367-370. [Medline] [CrossRef]

16) Endleman I, Critchley DJ: Transversus abdominis and obliquus internus activity during pilates exercises: measurement with ultrasound scanning. Arch Phys Med Rehabil, 2008, 89: 2205-2212. [Medline] [CrossRef]

17) Brill PW, Suffes S, Witz M, et al.: The Core Program: Fifteen Minutes a Day that Can Change Your Life. New York: Buntam Books. 2008, pp $1-231$.

18) Cho M: The influence of pelvic adjustment on the posture of female university students. J Phys Ther Sci, 2013, 25: 785-787. [Medline] [CrossRef] 